\title{
The Effect of Maternal Parenting Anxiety on Preschoolers' Self-Determination: Task Supportive Behaviors as Mediator
}

\author{
Hyun A Lee ${ }^{1}$, Kangyi Lee ${ }^{2}$ \\ M. A., Department of Interdisciplinary Program in Early Childhood Education, Seoul National University, Seoul, Korea ${ }^{1}$ \\ Professor, Department of Child Development and Family Studies, Seoul National University \& Adjunct Researcher, Research \\ Institute of Human Ecology, Seoul National University, Seoul, Korea ${ }^{2}$ \\ 어머니의 양육불안이 유아의 자기결정성에 미치는 영향: \\ 과제 지원 행동의 매개효과를 중심으로 \\ 이현아 ${ }^{1}$, 이강이 ${ }^{2}$ \\ 서울대학교 사범대학 협동과정 유아교육전공 석사 ${ }^{1}$, 서울대학교 아동가족학과 교수 및 서울대학교 생활과학연구소 겸임연구원 ${ }^{2}$
}

Objectives: The purpose of this study was to investigate the mediating effect of mother's task supportive behaviors on the relations between maternal parenting anxiety and preschoolers' selfdetermination.

Methods: Data were analyzed using frequency, mean, standard deviation, and multiple regression analysis with SPSS 25.0 program, PROCESS macro by Hayes (2013) was used to analyze the mediated effects.

Results: First, maternal parenting anxiety was negatively correlated to maternal cognitive scaffolding, maternal emotional scaffolding, and preschoolers' self-determination. However, it was positively correlated to maternal directive-controlling behaviors. Second, when controlling family income, maternal parenting anxiety and directive-controlling behaviors had a significant negative effect on preschooler's self-determination. Moreover, maternal emotional scaffolding had a significant positive effect on preschooler's self-determination. Lastly, the effects of maternal parenting anxiety on preschoolers' selfdetermination were partially mediated by maternal emotional scaffolding and maternal directivecontrolling behaviors.

Conclusion: This study confirms that maternal parenting anxiety and directive-controlling behaviors have a negative influence on preschooler's self-determination. Additionally, it is important to provide compassionate support such as praise and encouragement to foster the development of preschooler's self-determination.

Keywords: preschoolers' self-determination, maternal parenting anxiety, mothers' task supportive behaviors

\section{Introduction}

자기결정성(self-determination)은 개인이 결정의 주체가 되

Corresponding Author: Kangyi Lee, Professor, Department of Child Development and Family Studies, Seoul National University \& Adjunct Researcher, Research Institute of Human Ecology, Seoul National University, Seoul, Korea

E-mail: kangyil@snu.ac.k
어 자신이 하고 싶은 일을 스스로 선택하는 것을 의미한다 (Deci \& Ryan, 1985). 자기결정성은 자아존중감, 자아실현 및 자아개발과도 긍정적으로 관련이 있으며(Levesque, Zuehlke,

(C)The Korean Association of Child Studies

This is an Open Access article distributed under the terms of the Creative Commons Attribution Non-Commercial License (http:// creativecommons.org/licenses/by-nc/4.0) which permits unrestricted noncommercial use, distribution, and reproduction in any medium, provided the original work is properly cited. 
Stanek, \& Ryan, 2004) 의미감과 삶의 목적을 제공하여 전반적 인 삶의 만족도를 높인다(Ryan, Huta, \& Deci, 2008).

특히 유아기는 자기결정성 발달이 시작되는 중요한 시기이 다(Carlton \& Winsler, 1998). 유아는 만 3세가 되면 자기 인식, 자기 평가에 대한 능력이 발달하고(Barrett \& Morgan, 1995), 만 5 세에는 언어의 내면화와 이를 활용한 문제해결 능력이 향 상되면서 자기 규제자의 역할을 받아들이게 된다. 자기 규제 가 일정 수준 가능해지면 유아는 환경에 대한 통제력을 얻게 되고, 이는 유아의 유능감과 자율성을 촉진시켜 자기결정적 행동을 하게 된다(Carlton \& Winsler, 1998). 이에 국가 차원의 교육과정에서도 유아의 자율성 신장을 교육 목표로 두고 있다 (Ministry of Education, 2019), 누리과정의 각 영역에서는 유아 가 자유롭고 자발적으로 활동에 참여하도록 내용을 구성하였 으며, 이를 지원하는 것을 교사의 역할로 명시하였다.

자기결정성은 유아기 때부터 시작되는 발달 과정으로 간주 되며(Brotherson, Cook, Erwin, \& Weigel, 2008), 발달적으로 중 요한 함의를 가지고 있음에도 불구하고 아직까지 유아를 대상 으로 한 국내 연구는 소수에 불과하다. 국내의 자기결정성 연 구는 주로 학령기 아동을 대상으로만 한정적으로 이루어졌으 며 이들의 학업적인 부분에 중점을 두어 자기결정적 동기와 어떠한 관계가 있는지를 주로 다루어왔다(Choi \& Lew, 2010; H.-J. Ju, 2011; M. Kim \& Shin, 2010). 한편, 유아의 자기결정 성을 연구한 Y. J. Kim (2018), Park과 Seo (2013)의 연구에서는 주로 자기조절력이나 자아존중감과 같은 유아의 내재적 측면 과 자기결정성 관계에 초점을 맞추고 있다. 그러나 자기결정 적 행동을 하는 데 있어서 개인의 내적 자원뿐만 아니라 개인 을 둘러싼 환경 역시 매우 중요한 역할을 한다(Deci \& Ryan, 2000). 따라서 유아의 자기결정성 발달을 탐색함에 있어 유아 에게 의미 있는 환경을 고찰할 필요가 있다.

유아에게 상당한 영향력을 발휘하는 환경은 가족이며, 주 양육자인 어머니는 다른 어떠한 요인보다도 유아의 자기결 정성에 직접적인 영향을 미칠 수 있는 존재이다. 특히, 어머니 의 정서성은 유아의 발달에 영향을 주는 중요한 요인으로 인 식되어 왔다. 그 중 어머니의 부정적 정서는 유아의 인지를 비 롯한 사회.정서 발달 측면에서 중요하게 다루어져 왔다. 그동 안 국내외 연구들은 어머니의 부정적 정서 중에서 우울에 집 중하여 자녀 발달과의 관계를 살펴보았다(Hoffman, Crnic, \& Baker, 2006; A. Kim \& Doh, 2018; J. Lee \& Jang, 2009; Trapolini, McMahon, \& Ungerer, 2007). 그러나 어머니의 불안 역시 우울 만큼 자녀에게 미치는 영향력이 중대하며, 불안이 우울보다 사회적 단서를 위협적으로 해석하는데 일관성이 있다는 점과
인지적 왜곡을 개선시키는 효과가 더 크다는 점을 고려할 때 (Hallion \& Ruscio, 2011), 어머니의 불안에 주목할 필요가 있다.

불안은 스트레스나 위협적인 상황으로부터 초래되는 부정 적 정서 반응을 의미한다(Spielberger, 1972). 선행연구에서 어머 니의 불안은 자기결정성을 이루는 요소인 자율성, 유능성, 관 계성을 저해한다는 연구 결과가 일관되게 보고되었다(Diener \& Kim, 2004; Ginsburg \& Schlossberg, 2002; Hudson \& Rapee, 2001; Lim, 2019; Rubin, Coplan, \& Bowker, 2009; Whaley, Pinto, \& Sigman, 1999). 구체적으로 불안한 어머니는 자녀가 스스로 상황을 예측하고 경험할 수 있는 기회를 제한하기 때문에 자 녀는 낮은 수준의 자율성을 갖게 된다(Ginsburg \& Schlossberg, 2002; Whaley et al., 1999). 또 불안한 어머니의 자녀는 자신의 능력에 대해 낮은 기대와 성취동기를 갖는 것으로 나타났다 (Hudson \& Rapee, 2001; Rubin et al., 2009). 또한, 국내외 연구 에서 어머니의 불안은 타인과 좋은 관계를 형성하고 협력하는 능력인 사회적 유능감을 저해하는 결과를 보여주었다(Diener $\& \mathrm{Kim}, 2004 ; \mathrm{Lim}, 2019)$. 어머니의 불안은 자녀와의 불안정-저 항 애착(insecure-ambivalent attachment)과도 밀접한 관련이 있 기 때문에(Stevenson-Hinde, Shouldice, \& Chicot, 2011) 자녀의 사회적 관계 형성에도 부정적인 영향을 미칠 것임을 예측할 수 있다. 앞서 언급한 연구들은 일반적인 불안을 의미하는 특성불 안이나 상황에 대한 일시적 감정인 상태불안에 따른 영향력을 살펴보았다. 그러나 한 개인의 정서는 그가 속한 환경이나 문 화적 배경을 고려하여 이해되어야 한다. 따라서 이 연구는 한 국 사회의 문화적 특성이나 한국의 부모-자녀 관계 특성을 반 영한 불안인 양육불안을 중심으로 유아의 자기결정성에 미치 는 영향을 살펴보고자 한다.

양육불안이란 어머니가 자녀 양육을 수행하면서 느끼는 불 안을 의미한다(We, 2014). 한국사회는 시대의 흐름에 따라 가 족의 개념과 기능이 지속적으로 변화해 왔지만, 자녀 양육에 대한 어머니의 책임과 역할에는 큰 변화가 없으며 오히려 그 중요성이 강조되고 있다. 이에 따라 자녀 양육의 주 책임자인 어머니는 자신에게 부과되는 기대와 역할에 대해 심리적인 부 담을 경험하게 되고 양육불안감을 느끼게 될 것이다. 실제로 한국, 프랑스, 일본 어머니를 대상으로 실시한 양육불안 비교 연구에서 한국의 어머니가 두 나라의 어머니에 비해 양육불 안 수준이 높은 것을 확인할 수 있다(Kang, Chung, Yee, \& Kim, 2014; Kong \& Baek, 2017). 따라서 한국 사회라는 배경 안에서 어머니가 자녀를 양육하며 느끼는 불안감을 살펴보는 것은 더 의미가 있으며, 이를 구체적으로 탐색하는 연구가 요구된다.

아직까지 어머니의 양육불안과 유아의 자기결정성을 독립 
된 변수로 놓고 양자 간의 영향력을 살펴본 연구는 없다. 그러 나 앞서 언급한 연구에서 어머니의 일반적인 불안이 자녀의 자기결정성 하위요인에 선형적으로 부정적 영향을 미쳤다는 결과를 미루어 볼 때, 어머니의 양육불안 역시 유아의 자기결 정성에 부정적인 영향을 미칠 것으로 유추할 수 있다.

어머니의 불안은 어머니의 행동에도 영향을 준다. 개인 이 내면으로 경험하는 정서는 외적으로 표현하는 행동 양식 과 밀접한 관련이 있기 때문이다(Cumberland-Li, Eisenberg, Champion, Gershoff, \& Fabes, 2003). 선행연구에서 불안한 어머 니는 자녀에게 가르침을 주거나 도움이 되는 시도, 보조의 역 할을 하는 것에 있어서 불안하지 않은 어머니에 비해 낮은 수 준을 보였다(Woodruff-Borden, Morrow, Bourland, \& Cambron, 2002). 또한 자녀에게 따뜻한 지지 행동을 덜 하였다(Demertzis, 2007; Moore, Whaley, \& Sigman, 2004; We, 2014). 반면, 불안 한 어머니는 자녀를 과도하게 통제하려는 모습을 보였으며 (Demertzis, 2007; Moore et al., 2004), 비판적이고 거부적인 행 동을 많이 하는 것으로 밝혀졌다(Ginsburg, Grover, Cord, \& Ialongo, 2006; Ginsburg, Grover, \& Ialongo, 2005; Whaley et al., 1999). 그러나 일부 연구에서는 불안한 부모와 일반 부모들 사이에서 자녀 통제 행동에 어떠한 차이도 보이지 않았으며 (Turner, Beidel, Roberson-Nay, \& Tervo, 2003; Woodruff-Borden et al., 2002), 어머니의 불안이 높을수록 애정적 양육 태도를 보 이기도 하였다(Y.-S. Lee, 2017). 이를 통해 어머니의 불안이 어 머니의 행동에 반드시 부정적인 영향을 미치는 것은 아님을 알 수 있다. 이렇듯 선행연구에서 나타나는 상이한 연구 결과들을 보았을 때, 자녀 양육이라는 특수한 상황에서 느끼는 어머니의 불안은 어머니의 행동에 어떤 영향을 미치는지, 또 그 방향은 어떠한지에 대해 구체적으로 탐색할 필요가 있다.

한편, 유아의 자기결정성 발달에 있어 어머니의 행동적 요인 에도 주목할 필요가 있다. 앞서 살펴본 양육불안이 어머니 내면 의 정서적 특성이라면 어머니의 행동은 가시적이고 표면적인 특성이라고 할 수 있다. Ryan과 Deci (2000)는 아동은 어린 시기 부터 내재적 동기에서 차이를 보이고, 이러한 차이는 가정 내에 서의 경험에 따라 달라진다고 하였다. 이는 어머니의 행동이 유 아의 자기결정성과 밀접하게 관련이 있음을 시사한다. 어머니 의 지지와 격려는 아동의 자율성 동기를 증가시키며(Grolnick, Ryan, \& Deci, 1991), 아동에게 선택권을 제공하고 아동의 결 정을 인정하는 것은 내재적 동기를 강화시킨다(Bronstein, Ginsburg \& Herreral, 2005). 또 어머니의 자녀를 애정으로 대하 고 아동의 자율성을 지지해주는 행동은 아동으로 하여금 자율 성, 관계성, 유능성에 대한 욕구를 충족시키게 되어 자아 발전
과 자기결정적 행동을 촉진시킨다(Deci \& Ryan, 1991).

이와는 반대로 어머니가 규정을 만들고 이를 지키도록 강요하는 행동은 자녀의 자율성 및 유능성을 저하시켰으며 (Gottfried, Fleming, \& Gottfried, 1994; Grolnick et al., 1991) 부 모의 감시나 부정적 평가 역시 자녀의 내재적 동기의 훼손으 로 이어졌다(Harackiewicz, Manderlink, \& Sansone, 1984; H. Lee \& Park, 2020; Lepper \& Greene, 1975; Noh, Park, \& Chee, 2011). 이처럼 부모의 차갑고 지시적인 행동은 아동으로 하 여금 인정이나 승인을 위해서 자율성을 포기하게 하고(Roth, Assor, Niemiec, Ryan, \& Deci, 2009; Ryan \& Lynch, 1989) 이 는 결과적으로 자기결정적 행동을 감소시켜 다른 사람의 의 견이나 평가를 의식하는 사람으로 변하게 한다(Deci \& Ryan, 1985). 어머니의 통제적이고 지시적인 행동과 마찬가지로 어 머니의 거부적·적대적 행동 역시 자기결정성 발달에 있어 부 정적인 영향을 미치는 것으로 나타났다(Ginsburg et al., 2005; Ginsburg et al., 2006; Petti, Laird, Dodge, Bates, \& Criss, 2001). 한편, 최근 자기결정성이론의 연구에서 구조제공과 같은 인 지적 지지 행동에 대한 관심을 두기 시작하였다. 선행연구에 따르면 구조제공은 아동의 유능성을 촉진할 수 있는 환경을 제공하였으며(Farkas \& Grolnick, 2010; Grolnick et al., 2014; Vansteenkiste et al., 2012), 정보에 대한 피드백과 안내는 아이 의 동기와 적응에 관련이 있었다(Farkas \& Grolnick, 2010). 같 은 맥락에서 어머니나 교사의 인지적 피드백과 정보 제공은 아동의 자기결정적 동기에 긍정적 영향을 주었다(Grolnick \& Ryan, 1989; H. Lee \& Parks 2020; Vansteenkiste et al., 2012).

이상의 변인 간의 관계를 종합해보면, 어머니의 양육불안 과 인지적 지원, 정서적 지원, 통제·지시적 행동, 적대적 행동은 유아의 자기결정성에 영향을 미칠 것으로 예상된다. 그리고 어 머니의 행동은 어머니의 양육불안에 따라 달라질 것으로 예상 된다. 따라서 어머니의 행동이 양육불안과 유아의 자기결정성 의 관계에서 잠재된 매개변수로 고려될 수 있다. Belsky (1984) 는 양육 행동은 적어도 부분적으로는 어머니 개인의 과거 경험 으로 형성된 어머니의 특성에 의해 영향을 받을 수 있으며, 이 러한 행동은 결과적으로 아동 발달에 영향을 주게 된다고 하였 다. 즉, 어머니의 특성은 자녀에게 영향을 미치는 과정에서 어 머니의 행동을 매개한다. 따라서 어머니의 심리적 특성인 불 안은 행동을 매개로 유아의 자기결정성에 영향을 미침을 유추 할 수 있다. 선행연구에서 부모가 불안감이 있거나 자녀 양육 에 있어 유능감이 없다고 인식하는 경우 지시적 행동을 택하게 되어 유아가 환경을 스스로 통제한다고 느끼는 정도를 저해한 다고 밝히고 있다(Becker, Ginsburg, Domingues, \& Tein, 2010; 
Coleman \& Karraker, 2003). 그러나 Ginsburg 등(2005)의 연구에 서는 불안이 높은 부모일지라도 훈련 과정에서 자녀들의 의견 표현을 격려하거나 지지 및 수용하는 태도를 부여하는 경우, 자녀의 불안 정도가 더 낮았다고 보고하였다. 이와 같은 견지 에서, 어머니가 불안한 상태더라도 지지하는 행동을 하는 경우 에는 자녀의 자기결정성을 저해하지 않을 수 있을 것이다.

이제까지의 선행연구들은 어머니의 불안, 행동, 유아의 자 기결정성을 각각 단일 차원에서 검증되어 왔으므로 이들 간의 관계를 포괄적으로 해석하기에는 한계가 존재한다. 따라서 이 연구는 어머니의 양육불안이 어떠한 행동을 통해 유아의 자기 결정성에 영향을 미치는지 구체적이고 통합적인 검증을 하고 자 한다. 또한 이 연구는 과제를 제시하여 유아들의 발화와 행 동으로 드러난 자기결정성을 살펴보고 더불어 자녀가 과제를 수행하는 동안 이를 지원하는 어머니의 행동도 직접 관찰하고 평정하고자 한다. 이를 통해 설문지 측정이 갖는 방법론적 한 계를 극복하여 보다 객관적이고 명확한 설명을 제공할 수 있 을 것으로 기대한다.

이 연구에서 설정한 연구문제는 다음과 같다.

\section{연구문제 1}

어머니의 양육불안과 과제 지원 행동, 유아의 자기결정성은 유의한 관계가 있는가?

\section{연구문제 2}

어머니의 양육불안과 과제 지원 행동은 유아의 자기결정성에 유의한 영향을 미치는가?

\section{연구문제 3}

어머니의 양육불안이 유아의 자기결정성에 미치는 영향을 과 제 지원 행동이 매개하는가?

\section{Methods}

\section{연구대상}

서울, 경기 지역에 위치한 유치원 및 어린이집 7곳, 보건소, 소 아과 병원, 관공서, 온라인 육아 카페 게시판에 모집문건을 게 시하여 자발적으로 참여를 희망한 79 명의 어머니와 그들의 만 5세 자녀, 총 158 명을 대상으로 자료를 수집하였다. 이 연 구의 과제는 시공간 능력을 요구하는 과제이므로, 유아의 시
각-공간적 처리와 부분 및 전체의 관계를 통합하는 능력이 높 거나 낮을 시 자기결정성 측정이 왜곡될 가능성이 있다. 따라 서 한국형 유아지능검사 4판(K-WPPSI-IV)의 시공간지표 검 사를 수행하여 상위 $2.2 \%$ 와 하위 $2.2 \%$ 에 해당하는 유아를 제 외하였다. 그 결과 시공간능력 상위 $2.2 \%$ 에 해당하는 유아 4 명과 과제 수행을 거부하거나 이탈한 유아 5명을 포함하여 총 9명의 유아가 제외되었으며 최종적으로 70 쌍의 유아-어머니 (총 140 명)가 연구에 참여하였다.

연구대상의 사회인구학적 특성은 다음 Table 1 에 제시하였 다. 어머니의 학력은 4 년제 대학교 졸업이 40 명 $(57.1 \%)$ 으로 가장 많았으며, 가정의 월 평균 소득수준은 600 만 원 이상이 46명(65.7\%)으로 가장 많았다. 자료수집이 진행되었던 2020 년 1분기 가구당 월평균 소득이 535만 8천 원임을 고려했을 때(Statistics Korea, 2020), 연구대상 유아 가정의 대부분은 중산 층 범위에 속한다고 볼 수 있다.

\section{연구도구}

$$
\text { 유아의 자기결정성 }
$$

이 연구에서는 자기결정성을 만 5세 유아가 과제를 수행하 는 동안 언어적 · 비언어적 행동으로 보인 자율성, 유능성, 관 계성으로 정의하였으며, 유아의 자기결정성을 측정하기 위해 Zhang과 Whitebread (2019)가 개발한 Children's Observed Needs Satisfaction을 번안.수정하여 사용하였다. 이 도구는 과제 수행 에서 보이는 유아의 언어적 - 비언어적 행동을 기준으로 유아의 자율성, 유능성, 관계성을 평정한다. 구체적으로 자율성은 유아 가 자유롭게 행동을 주도하고 조절할 수 있는 정도 및 유아가 문제해결 방법을 스스로 선택하는 정도로 평가한다. 자율성이 명백히 없이 어머니에게 완전히 통제되어 자신의 방식으로 상 황에 접근할 수 없다면 1점, 상당한 정도의 자율성을 보이며 과 제 이행에 있어서 지속적으로 자유롭게 선택할 수 있다면 5점 을 부여한다. 유능성은 지속성과 높은 상관관계를 갖는다는 선 행 연구(Schunk 1991; Zhang \& Whitebread, 2019)를 바탕으로 문제가 생겼을 때 유아가 과제 수행을 지속하는 정도로 판단한 다. 문제가 생겼을 때 작업을 즉시 중지하고 어머니와도 계속해 서 과제를 완료하지 않는 경우는 1점, 과제 전체에 걸쳐 어머니 와 함께 해결책을 찾을 때까지 계속해서 지속성을 보여주는 경 우에는 5점에 해당한다. 관계성은 어머니를 향해 긍정적인 감 정을 나타내는 정도(미소, 웃음, 목소리 톤, 긍정적인 바디 랭귀 지와 언어)로 판단한다. 어머니를 향한 긍정적인 감정 표현이 
Table 1

Demographic Information of Participants

\begin{tabular}{lccc}
\hline \multicolumn{1}{c}{ Participants } & $N(\%)$ & Age range (Month) & Mean age (Month) \\
\hline Boys & $28(40.0)$ & $71-84$ & 76.93 \\
Girls & $42(60.0)$ & $72-83$ & 76.48 \\
Mothers & $70(100.0)$ & $31-45$ & 37.89 \\
\hline \multicolumn{2}{c}{ Variables } & Categories & $N(\%)$ \\
\hline Maternal academoc background & Under college graduate & $20(28.6)$ \\
& & University graduate & $40(57.1)$ \\
& Graduate School & $10(14.3)$ \\
\hline Monthly household income (10,000won) & Less than 399 & $10(14.3)$ \\
& Over 400-599 & $14(20.0)$ \\
\end{tabular}

전혀 없다면 1점, 어머니를 향한 긍정적인 표현이 상호작용 절 반 이상에 걸쳐 표현하는 경우에는 5점을 부여한다. 자기결정 성 측정을 위해 종이접기와 펜토미노 순으로 과제를 제시하였 다. 과제의 수행시간은 각각 10 분이다. 유아가 종이접기와 펜 토미노 과제를 수행하는 모든 과정은 연구대상자들의 동의하 에 녹화하였으며, 연구자는 녹화된 영상을 보고 유아의 자율성, 유능성, 관계성을 평정하였다. Zhang과 Whitebread (2019)의 도 구는 1점부터 5점까지 측정하는 5점 Likert 척도이며 점수가 높 을수록 각 하위 항목이 높음을 의미한다. Zhang과 Whitebread (2019)의 연구를 바탕으로 2 분을 분석 단위로 정하였다. 각각의 하위 항목을 2 분 단위로 평정한 뒤 평균값을 산출하고 하위항 목을 합산하여 자기결정성 점수를 산출하였다. 과제 1 과 2 의 자 기결정성 평균점수를 이용하여 최종적으로 유아의 자기결정성 변수로 사용하였다.

\section{과제 지원 행동}

어머니의 과제 지원 행동은 자녀가 종이접기와 펜토미노 총 두 개의 과제를 수행하는 동안 어머니의 언어적.비언어적 행동에 초점을 맞추어 평가하였다. 이를 위해 Cha (2015)의 Maternal Scaffolding 코딩 매뉴얼을 번안.수정하여 사용하였다. Cha (2015)는 선행연구를 바탕으로 과제 상황에서 어머니의 행동을 인지적 비계설정, 정서적 비계설정, 지시적 · 통제적 행 동, 거부적 - 적대적 행동 4 가지 범주로 구분하였다. 인지적 비 계설정은 과제를 체계화하는 방법이나 문제 해결에 필요한 전 략 포함된 정보를 제공하는 것과 같은 정교화된 발화, 아동이 스스로 처리 가능한 수준의 간단한 설명을 제공하는 난이도 조절, 과제의 진행상황을 검토하거나 최종 목표를 재확인하는
확인 및 검토로 구성되어 있다. 정서적 비계설정은 칭찬이나 격려, 심정이나 의도를 알아주는 말, 유아의 의사에 대해 물어 보는 등 온정적지지 환경을 제공해주는 행동을 뜻한다. 지시 적·통제적 행동은 과제를 수행중인 유아에게 무엇을 할지 지 시를 하거나 자녀에게 필요한 도움 수준을 넘어 어머니가 직 접 과제를 해결하는 행동을 의미한다. 거부적.적대적 행동은 유아가 도움을 요청하는 사인이나 발언을 무시하거나 거부하 기, 비꼬기, 비난하기, 제지하기 등의 행동이 포함된다. 이 연 구에서는 위의 4 가지 범주에 속하지 않은 행동을 기타 범주로 구분하여 과제 수행 동안 어머니의 전체 행동을 분석하였다. 자녀의 과제 수행 동안 녹화한 영상을 모두 전사하여 전사본 을 토대로 어머니 행동의 빈도를 파악하고, 전체 행동에서 각 하위 행동에 대한 비율을 계산하여 분석하였다.

\section{어머니의 양육불안}

어머니의 양육불안을 측정하기 위해 We (2014)가 제작하고 타당도를 검증한 양육불안척도를 사용하였다. 총 26문항으로 5 개의 하위 요인(부모역할 효능감으로 인한 불안, 자녀와의 애착에 대한 불안, 자녀에 대한 염려, 사회적 지지에 대한 염 려, 완벽주의로 인한 불안)을 측정한다. 부모역할 효능감으로 인한 불안은 "나는 부모로서 아이에게 좋은 본보기를 보여주 지 못할까봐 걱정이 된다.” 등이 있으며, 자녀와의 애착에 대 한 불안은 “나는 나의 부모님에게 따뜻하거나 지지를 받아본 경험이 없어서, 내 아이와 감정과 생각을 나누는 것이 불편하 다." 등이 있다. 자녀에 대한 염려는 "나는 아이가 남들만큼 잘 하지 못하면 뒤떨어진 사람이 될 것 같다.” 등이 있으며, 사회 적 지지에 대한 염려는 "양육에 대해서 주변 사람들에게 도움 
을 받는 것은 신뢰할 수 없다.” 등이 있다. 마지막으로 완벽주 의로 인한 불안은 "주변 사람들은 내가 부모로서 완벽주의 성 향이 있다고 한다.", "나는 부모로서 꼭 성공해야만 한다." 등 이 있다. 5점 Likert식 평정척도이며, 양육불안의 점수는 모든 문항의 평균점수를 사용하였다. 점수가 높을수록 양육불안이 높음을 의미한다. 양육불안 전체의 신뢰도 계수(Cronbach's $\alpha$ ) 는 .90으로 나타났다.

\section{연구절차}

\section{예비조사와 본조사}

본 연구의 과제 및 연구 도구의 적합성 검증을 위하여 약 20 일 간 서울대학교 아동행동 관찰실에서 7쌍의 유아(여아 5명, 남 아 2명)와 어머니를 대상으로 예비조사를 실시하였다. 본조사 는 2019년 11월 20일부터 2020년 1월 19일까지 서울시와 경 기도 소재의 유치원과 어린이집 7곳과 서울대학교 아동행동 관찰실에서 만 5세 유아와 유아의 어머니 총 79쌍(158명)을 대 상으로 실시하였다.

\section{평정자 훈련}

유아의 자기결정성과 어머니의 행동을 분석하기 위하여 아동 학.유아교육학 박사과정에 재학 중인 2 명의 대학원생과 연구 자 본인으로 구성된 3 인의 평정자가 평정척도에 대해 숙지하 고 5사례를 무선으로 선정하여 녹화된 영상을 보며 평정실습 을 하였다. 총 9 회에 걸쳐 약 45 시간 동안 각 평정 척도의 의미 와 기준에 대해 논의를 한 후 최종적으로 평정 매뉴얼을 완성 하였다. 이후 무선으로 선정한 10 사례를 각자 평정한 후 관찰 자 간 일치도를 분석하기 위해 급내상관계수(ICC)를 산출하 였다. 유아의 자기결정성에 대한 급내상관계수(ICC)는 유능 성이 .85 , 자율성이 .94 , 관계성이 .79였으며 어머니의 행동에 대한 평정자 간 일치도는 인지적 비계설정이 .92, 정서적 비계 설정이 .98, 지시적·통제적 행동이 .94, 거부적·적대적 행동 이 .96이었다.

\section{자료분석}

수집된 자료는 IBM SPSS 25.0 (IBM Co., Armonk, NY)과 PROCESS macro version 3.5 (Hayes, 2013) 프로그램을 사용하 여 분석하였다. 통계적 방법으로는 기술통계, 일원분산분석 (one-way ANOVA), Pearson의 적률상관분석, 다중회귀분석, 매 개효과 검증을 사용하였다.

\section{Results}

\section{어머니의 양육불안, 과제 지원 행동, 유아의 자기결정성의 관계(연구문제 1)}

연구문제별 분석에 앞서 주요변수의 기술통계를 실시한 결 과는 다음과 같다. 어머니 양육불안의 평균 점수는 $2.48(S D=$ .51)점으로 나타나 보통 수준임을 알 수 있다. 어머니는 과제 상황에서 지시적 · 통제적 행동을 가장 많이 보였으며, 다음으 로 인지적 비계설정, 정서적 비계설정, 적대적·거부적 행동 순으로 행동하였다. 유아의 자기결정성 평균 점수는 3.33 (SD $=.46)$ 점으로 나타나 보통 수준임을 확인하였다.

다음으로 주요 변인이 어머니의 학력과 가구소득에 따라 유의한 차이를 보이는지 살펴보기 위해 일원분산분석을 실시 하였다. Table 2에서 보여지는 바와 같이 가구소득에 따라서 부분적으로 유의한 차이를 보였다. 구체적으로 어머니의 불 안은 가구소득이 600만 원 이상일 때보다 399미만인 경우 더 높은 것으로 나타났다. 그러나 인지적 비계설정은 가구소득 이 399미만인 경우보다 600 만 원 이상일 때 더 많이 하는 것 으로 확인되었다. 이는 사회경제적 수준이 높은 부모는 자녀 의 문제해결을 보다 효과적으로 지원하는 데 도움이 되는 지 적 자원을 가지고 있기 때문에 자녀에게 인지적 지원을 제공 하는 과정에서 더 숙련된 전략을 사용한다(Neitzel, Dopkins, \& Stright, 2004)는 선행연구와 맥을 같이하는 부분이다.

연구문제 1 을 알아보기 위해 Pearson의 적률상관분석을 실 시한 결과는 Table 3 과 같다. 주요 변인 간 부분적으로 유의한 상관이 있는 것으로 나타났다. 주요변인과 상관관계가 유의하 지 않은 거부적.적대적 행동은 이후 분석에서 제외한다.

\section{어머니의 양육불안과 과제 지원 행동이 유아의 자기결정성에 미치는 영향(연구문제 2)}

어머니의 양육불안과 어머니의 과제 지원 행동이 유아의 자 기결정성에 미치는 영향이 어떠한지 알아보기 위하여 다중회 귀분석을 실시하였다. 앞서 가구소득이 주요 변인과 부분적 인 차이를 보였으므로 가구소득을 통제변인, 어머니의 양육 불안과 어머니의 과제 지원 행동을 독립변인, 유아의 자기결 
Table 2

Differences in Variables According to Maternal Academic Background and Monthly Household Income

\begin{tabular}{|c|c|c|c|c|c|c|c|c|c|}
\hline & \multicolumn{3}{|c|}{ Maternal academic background } & \multirow[b]{2}{*}{$F$} & \multicolumn{3}{|c|}{ Monthly household income } & \multirow[b]{2}{*}{$F$} & \multirow[b]{2}{*}{$M(S D)$} \\
\hline & $\begin{array}{l}\text { Under } \\
\text { College } \\
M(S D)\end{array}$ & $\begin{array}{l}\text { University } \\
M(S D)\end{array}$ & $\begin{array}{c}\text { Graduate } \\
\text { school } \\
M(S D)\end{array}$ & & $\begin{array}{c}\text { Less than } \\
399 \\
M(S D)\end{array}$ & $\begin{array}{c}\text { Over } 400- \\
599 \\
M(S D)\end{array}$ & $\begin{array}{c}\text { More than } \\
600 \\
M(S D)\end{array}$ & & \\
\hline Maternal parenting anxiety & $\begin{array}{l}2.62 \\
(.52)\end{array}$ & $\begin{array}{l}2.49 \\
(.48)\end{array}$ & $\begin{array}{c}2.18 \\
(.55)\end{array}$ & 2.46 & $\begin{array}{c}2.98 b \\
(0.39)\end{array}$ & $\begin{array}{l}2.70 \mathrm{ab} \\
(.45)\end{array}$ & $\begin{array}{c}2.31 \mathrm{a} \\
(.46)\end{array}$ & $11.41^{* * *}$ & $\begin{array}{l}2.48 \\
(.51)\end{array}$ \\
\hline Cognitive scaffolding & $\begin{array}{c}23.14 \\
(5.79)\end{array}$ & $\begin{array}{c}23.62 \\
(8.17)\end{array}$ & $\begin{array}{l}25.51 \\
(3.38)\end{array}$ & .39 & $\begin{array}{c}18.96 a \\
(6.81)\end{array}$ & $\begin{array}{l}22.10 \mathrm{ab} \\
(6.99)\end{array}$ & $\begin{array}{c}25.29 \mathrm{~b} \\
(6.58)\end{array}$ & $4.21^{*}$ & $\begin{array}{c}23.75 \\
(7.00)\end{array}$ \\
\hline Emotional scaffolding & $\begin{array}{c}16.59 \\
(5.20)\end{array}$ & $\begin{array}{l}17.06 \\
(7.29)\end{array}$ & $\begin{array}{l}19.74 \\
(6.60)\end{array}$ & .81 & $\begin{array}{l}16.95 \\
(4.17)\end{array}$ & $\begin{array}{l}14.79 \\
(6.32)\end{array}$ & $\begin{array}{l}18.15 \\
(7.07)\end{array}$ & 1.41 & $\begin{array}{c}17.31 \\
(6.65)\end{array}$ \\
\hline Directive.controlling behaviors & $\begin{array}{l}32.16 \\
(8.86)\end{array}$ & $\begin{array}{c}27.85 \\
(10.99)\end{array}$ & $\begin{array}{c}24.49 \\
(10.46)\end{array}$ & 2.07 & $\begin{array}{l}29.65 \\
(8.71)\end{array}$ & $\begin{array}{c}34.55 \\
(13.74)\end{array}$ & $\begin{array}{l}26.56 \\
(9.19)\end{array}$ & $3.37^{*}$ & $\begin{array}{c}28.60 \\
(10.52)\end{array}$ \\
\hline Hostile behaviors & $\begin{array}{c}6.80 \\
(4.92)\end{array}$ & $\begin{array}{c}4.40 \\
(3.16)\end{array}$ & $\begin{array}{c}3.91 \\
(4.16)\end{array}$ & 3.05 & $\begin{array}{c}4.27 \\
(3.78)\end{array}$ & $\begin{array}{c}5.49 \\
(3.98)\end{array}$ & $\begin{array}{c}5.03 \\
(4.09)\end{array}$ & .27 & $\begin{array}{c}5.02 \\
(3.98)\end{array}$ \\
\hline
\end{tabular}

Note. Scheffe's Test.

${ }^{*} p<.05 .{ }^{* *} p<.01 .{ }^{* * *} p<.001$.

Table 3

Correlations Among Variables

\begin{tabular}{|c|c|c|c|c|c|c|c|}
\hline & ables & 1 & 2 & 3 & 4 & 5 & 6 \\
\hline 1. Maternal parenting anxiety & & - & & & & & \\
\hline \multirow[t]{3}{*}{ Mothers' task supportive behaviors } & 2. Cognitive scaffolding & $-.57^{* *}$ & - & & & & \\
\hline & 4. Directive.controlling behaviors & $.41^{* *}$ & -.23 & $-.66^{* *}$ & - & & \\
\hline & 5. Hostile behaviors & .23 & $-.24^{*}$ & -.20 & -.10 & - & \\
\hline
\end{tabular}

Note. $N=70$.

${ }^{*} p<.05 .{ }^{* *} p<.01$.

정성을 종속변인으로 설정하였다. 분석에 앞서 분산팽창계수 (VIF), Durbin-Watson 지수를 조사하였으며, 모두 기본 가정에 충족하는 값을 보였다.

구체적인 연구결과는 다음 Table 4 와 같다. 어머니의 양육불 안 $(\beta=-.43, p<.001)$, 정서적 비계설정 $(\beta=.22, p<.01)$ 과 지시적. 통제적 행동 $(\beta=-.38, p<.01)$ 이 종속변수에 대해 유의한 영향력 을 갖는 것으로 나타났으며, 이들 변수에 의한 종속변수의 총 설명량은 $61 \%$ 이었다 $(F=20.22, p<.001)$. 이러한 결과는 어머니 의 양육불안이 낮을수록 정서적 비계설정을 많이 할수록 지시 적·통제적 행동을 적게 할수록 과제 상황에서 보이는 유아의 자 기결정성이 높아지는 것을 의미한다. 그러나 인지적 비계설정 의 경우, 과제 상황에서 보이는 유아의 자기결정성에 유의한 영 향을 미치지 않는 것으로 나타났다 $(\beta=-.09, n s)$.

\section{어머니의 양육불안과 유아의 자기결정성 관계 에서 과제 지원 행동의 매개효과(연구문제 3)}

연구문제 3 에서는 유아의 자기결정성에 유의한 영향을 미친 정서적 비계설정과 지시적·통제적 행동을 중심으로 매개효과 를 살펴보고자 한다.

어머니의 양육불안과 유아의 자기결정성의 관계 에서 정서적 비계설정의 매개효과

독립변인인 어머니의 양육불안이 매개변인인 정서적 비계설 정에 미치는 영향에 대한 회귀모형은 $13 \%$ 의 설명력을 보였으 며 통계적으로 유의하였다 $(F=5.04, p<.01)$. 양육불안이 정서 
Table 4

Multiple Regression Analysis: Effect of Maternal Parenting Anxiety and Mothers' Task Supportive Behaviors on Preschoolers' Self-Determination

\begin{tabular}{|c|c|c|c|c|c|}
\hline Variable & $B$ & $S E$ & $\beta$ & $R^{2}$ & $F$ \\
\hline Monthly household income & -.03 & 0.06 & -.04 & \multirow[t]{2}{*}{.61} & \multirow[t]{2}{*}{$20.22^{* * *}$} \\
\hline Maternal parenting anxiety & -.38 & 0.10 & $-.43^{* * *}$ & & \\
\hline \multicolumn{6}{|l|}{ Mothers' task supportive behaviors } \\
\hline Cognitive scaffolding & -.01 & 0.01 & -.09 & & \\
\hline Emotional scaffolding & .02 & 0.01 & $.22^{* *}$ & & \\
\hline Directive.controlling behaviors & -.02 & 0.00 & $-.38^{* * *}$ & & \\
\hline
\end{tabular}

${ }^{* *} p<.01 .{ }^{* * *} p<.001$.

Table 5

The Mediating Effect of Emotional Scaffolding

\begin{tabular}{lllccccc}
\hline \multicolumn{1}{c}{ Independent variable } & & & Dependent variables & $\beta$ & $S E$ & $t$ & $R^{2}$ \\
\hline MPA & $\rightarrow$ & Emotional scaffolding & $-5.08^{* *}$ & 1.71 & -2.98 & .13 & $5.04^{* *}$ \\
MPA, Emotional scaffolding & $\rightarrow$ & Preschoolers' self-determination & $-.39^{* * *}$ & .09 & -4.28 & .53 & $25.20^{* * *}$ \\
& & & $.03^{* * *}$ & .01 & 5.11 & .53 & $25.20^{* * *}$ \\
\hline
\end{tabular}

Note. $N=70 . \mathrm{MPA}=$ maternal parenting anxiety.

${ }^{* *} p<.01 .{ }^{* * *} p<.001$.

Table 6

Bootstrapping Method test of the Indirect Effect of Emotional Scaffolding on the Relationship Between Maternal Parenting Anxiety and Preschoolers' Self-Determination

\begin{tabular}{|c|c|c|c|c|}
\hline \multirow[b]{2}{*}{ Path } & \multirow[b]{2}{*}{$\beta$} & \multirow[b]{2}{*}{$S E$} & \multicolumn{2}{|c|}{$95 \% \mathrm{CI}$} \\
\hline & & & LLCI & ULCI \\
\hline Maternal parenting anxiety $\rightarrow$ Emotional scaffolding $\rightarrow$ Preschoolers' self-determination & -.16 & .06 & -.30 & -.05 \\
\hline
\end{tabular}

적 비계설정에 미치는 영향은 통계적으로 유의한 부적 영향을 미치는 것으로 나타났다 $(\beta=-5.08, p<.01)$. 어머니의 양육불 안과 정서적 비계설정이 자기결정성에 미치는 영향에 대한 회 귀 모형은 $53 \%$ 의 설명력을 보였으며 통계적으로 유의하였다 $(F=25.20, p<.001)$. 어머니의 양육불안은 유아의 자기결정성 에 통계적으로 유의한 부적 영향을 미쳤고 $(\beta=-.39, p<.001)$, 어머니의 정서적 비계설정은 유아의 자기결정성에 유의한 정 적 영향을 미쳤다 $(\beta=.03, p<.001)$. 이는 어머니의 양육불안 이 높으면 어머니의 정서적 비계설정과 유아의 자기결정성이 낮아지고, 어머니의 정서적 비계설정이 높으면 유아의 자기결 정성이 높아짐을 의미한다.

정서적 비계설정의 매개효과 유의성을 검증하기 위해 PROCESS macro 부트스트래핑 분석을 실시하였다. 이 매개경 로 $($ 양육불안 $\rightarrow$ 정서적 비계설정 $\rightarrow$ 자기결정성 $)$ 는 $95 \%$ 신뢰 구간(-.30 -.05)에 0이 포함되지 않았으며 모두 0보다 작기 때 문에 매개효과가 부적으로 유의한 것을 알 수 있다.

\section{어머니의 양육불안과 유아의 자기결정성의 관계에서 지시적·통제적 행동의 매개효과}

Table 7에서 제시한 바와 같이, 어머니의 양육불안이 지시 적 · 통제적 행동에 미치는 영향에 대한 회귀모형은 $17 \%$ 의 설 명력을 보였으며 통계적으로 유의하였다 $(F=6.71, p<.01)$. 양육불안이 지시적·통제적 행동에 미치는 영향은 통계적으 로 유의한 정적 영향을 미치는 것으로 나타났다 $(\beta=8.51, p<$ .01). 어머니의 양육불안과 지시적.통제적 행동이 자기결정성 에 미치는 영향에 대한 회귀 모형은 $57 \%$ 의 설명력을 보였으 며 통계적으로 유의하였다 $(F=29.73, p<.001)$. 어머니의 양 육불안은 유아의 자기결정성에 통계적으로 유의한 부적 영향 을 미쳤고 $(\beta=-.36, p<.001)$, 어머니의 지시적 · 통제적 행동은 유아의 자기결정성에 유의한 부적 영향을 미쳤다 $(\beta=-.02, p$ <.001). 이는 어머니의 양육불안이 높으면 지시적·통제적 행 동은 높아지고 유아의 자기결정성은 낮아지며, 어머니의 지시 
Table 7

The Mediating Effect of Directive. Controlling Behaviors

\begin{tabular}{llcrrrrr}
\hline \multicolumn{1}{c}{ Independent variable } & & Dependent variables & $\beta$ & \multicolumn{1}{c}{$S E$} & \multicolumn{1}{c}{$R^{2}$} \\
\hline MPA & $\rightarrow$ & DCB & $8.51^{* *}$ & 2.64 & 3.22 & .17 & $6.71^{* *}$ \\
MPA, DCB & $\rightarrow$ & Preschoolers' self-determination & $-.36^{* * *}$ & .09 & -4.09 & .57 & $29.73^{* * *}$ \\
MPA, DCB & $\rightarrow$ & Preschoolers' self-determination & $-.02^{* * *}$ & .00 & -5.91 & $29.73^{* * *}$ \\
\hline
\end{tabular}

Note. $N=70 . \mathrm{MPA}=$ maternal parenting anxiety; $\mathrm{DCB}=$ directive $\cdot$ controlling behaviors.

${ }^{* *} p<.01 .{ }^{* * *} \mathrm{p}<.001$.

Table 8

Bootstrapping Method test of the Indirect Effect of Directive. Controlling Behaviors on the Relationship Between Maternal Parenting Anxiety and Preschoolers' Self-Determination

\begin{tabular}{|c|c|c|c|c|}
\hline \multirow[b]{2}{*}{ Path } & \multirow[b]{2}{*}{$\beta$} & \multirow[b]{2}{*}{$S E$} & \multicolumn{2}{|c|}{$95 \% \mathrm{CI}$} \\
\hline & & & LLCI & ULCI \\
\hline Maternal parenting anxiety $\rightarrow$ Directive.controlling behaviors $\rightarrow$ Preschoolers' self-deter & -.19 & .06 & -.33 & -.09 \\
\hline
\end{tabular}

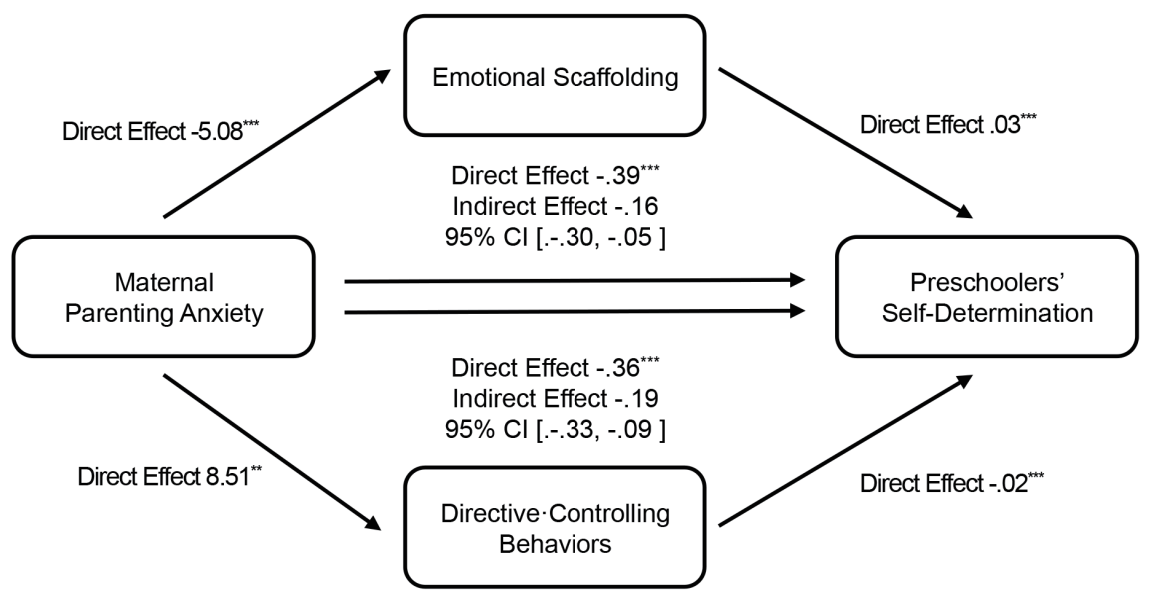

Figure 1. Mediating effects of emotional scaffolding and directive-controlling behaviors on the relationship between maternal parenting anxiety and preschoolers' self-determination.

${ }^{* *} p<.01 .{ }^{* * *} p<.001$.

적·통제적이 높으면 유아의 자기결정성이 낮아짐을 의미한다.

어머니 지시적·통제적 행동의 매개효과 유의성을 검증하기 위해 PROCESS macro 부트스트래핑 분석을 실시한 결과, 이 매 개경로(양육불안 $\rightarrow$ 지시적 · 통제적 행동 $\rightarrow$ 유아의 자기결정 성)는 95\% 신뢰구간(-.33 -.09)에 0이 포함되지 않아 매개효과 가 통계적으로 유의한 것을 알 수 있다(Table 8 참조). 어머니의 양육불안과 유아의 자기결정성의 관계에서 정서적 비계설정과 지시적·통제적 행동의 매개효과 도식은 Figure 1과 같다.

\section{Discussion}

이 연구는 유아의 자기결정성 발달에 있어 어머니가 중요한
환경적 맥락이 되는 점을 고려하여 어머니의 심리적인 요인인 양육불안과 표면적으로 드러나는 어머니 과제 지원 행동에 주 목하였다. 어머니의 심리적 특성과 행동적 특성은 서로 유기 적으로 연계되어 유아의 발달에 영향을 미침에도 불구하고 선 행연구에서는 자기결정성에 영향을 미치는 어머니의 불안과 행동을 분절적으로 살펴보았다. 이에 본 연구에서는 유아에게 과제를 제시하여 어머니의 양육불안이 유아의 자기결정성에 미치는 영향을 살펴보고 어머니의 과제 지원 행동이 이를 매 개하는지를 검증하여 유아의 자기결정성 발달에 대한 보다 명 확한 이해 및 관련 시사점을 도출하고자 하였다.

분석 결과를 토대로 다음과 같은 결론을 도출하였다. 첫 째, 주요 변인들은 서로 유의미한 상관관계를 보였지만, 거부 
적.적대적 행동은 주요변인들과의 상관관계가 유의하지 않았 다. 이는 어머니의 불안과 거부적 행동(Ginsburg et al., 2005; Ginsburg et al., 2006; Whaley et al., 1999), 거부적 행동과 유아 의 자율성, 유능성, 관계성의 부적 관계를 보고한 선행연구와 는 다른 결과이다(Ginsburg et al., 2005; Ginsburg et al., 2006). 이 연구는 자연스러운 놀이 상황이 아닌 과제가 주어진 관찰 상황에서 이루어졌고, 자신의 행동이 녹화되어 기록되고 있다 는 것을 인식한 어머니가 의식적으로 거부적·적대적 행동을 덜 했을 개연성이 존재한다. 이에 따라 거부적.적대적 행동이 바닥효과를 보여 이와 같은 결과를 보였을 가능성이 있다.

둘째, 어머니의 양육불안은 과제 수행 중 관찰된 유아의 자 기결정성에 유의한 부적 영향을 미쳤다. 이는 어머니의 불안이 유아의 자율성, 유능성, 관계성(Diener \& Kim, 2004; Ginsburg \& Schlossberg, 2002; Lim, 2019; Rubin et al., 2009; Whaley et al., 1999)에 부정적인 영향을 미친다는 선행연구를 지지하는 결과 로, 상위 요인인 자기결정성에도 동일한 영향을 미치는 것을 확인할 수 있었다. 이러한 결과는 어머니의 양육불안이 자녀의 자기결정성을 저해하므로 양육불안을 감소시킬 수 있는 적절 한 지원이 필요함을 시사한다. 특히, 만 5세 자녀를 둔 어머니 를 대상으로 양육불안을 감소시키는 지원이 필요할 것으로 사 료된다. 아동이 학교생활에 원만하게 적응하기 위해서는 자기 결정성 함양이 필요하다(Grolnick et al., 1991; Yeo, 2015). 만 5세 는 자기결정성 발달에 중요한 시기임과 동시에 여러 과제를 수 행해야 하는 초등학교 진입을 앞두고 있으므로 자기결정성 함 양을 위한 지원이 더욱 요구된다. 따라서 이후 아동의 성공적 인 학교 적응을 위해 만 5 세 자녀를 둔 어머니의 양육불안감을 해소시키는 노력이 선행되어야 할 것이다.

한편, 자녀가 과제를 수행할 때 어머니가 제공한 인지적 비 계설정은 유아의 자기결정성에 유의한 영향을 미치지 못했 다. 이는 아동의 자기결정적 동기에 있어 어머니나 교사의 인 지적 피드백과 정보 제공이 긍정적 영향을 준다는 선행 연구 (Grolnick \& Ryan, 1989; Vansteenkiste et al., 2012)와 다른 결과 이다. Grolnick 등(2014)은 구조 제공과 같은 인지적 비계설정 은 아동이 과제의 주도권을 느끼게 하는 정도를 감소시킬 수 있음을 지적하였는데, 이 같은 맥락을 고려해보면 어머니가 정보를 제공해주거나 과제 상황을 확인해주는 행동은 유아로 하여금 자신 행동에 대한 소유권을 침해받았다고 느끼게 하여 유아의 자기결정성에 영향을 미쳤을 가능성을 제기할 수 있 다. 따라서 유아의 자기결정성 발달을 위한 어머니의 인지적 지지 행동은 유아가 자신의 자율성이 지지된다고 느끼는 수준 안에서 구현되어야 할 것이다.
셋째, 어머니 과제 지원 행동의 매개 효과 여부를 검토한 결 과, 어머니의 양육불안은 정서적 비계설정 행동을 통해 유아 의 자기결정성에 간접적인 영향을 미치는 것으로 나타났다. 이와 같은 결과는 부모의 지지와 격려가 자율적 동기를 증가 시킨다는 선행연구(Deci \& Ryan, 1991; Grolnick et al., 1991; Sung, Bang, \& Lee, 2005; Tafarodi, Milne, \& Smith, 1999)를 결 과를 재확인 시켜주었다. 또한 Ginsburg 등(2005)이 불안한 부 모일지라도 자녀들을 격려하거나 지지하는 태도를 보일 경우 자녀의 불안 정도가 더 낮았다고 보고한 것과 맥을 같이하는 결과로, 어머니의 양육불안감이 높더라도 자녀가 과제를 할 때 어머니가 제공하는 정서적 비계설정을 통해 유아의 자기결 정성이 지지될 수 있음을 시사한다. 따라서 유아의 자기결정 성 발달에 있어서 어머니의 따뜻하고 온정적인 지지 행동의 중요성이 부각된다.

마지막으로 어머니의 지시적·통제적 행동은 양육불안과 유 아의 자기결정성 관계에서 부분 매개 역할을 하는 것으로 나 타났다. 이와 같은 결과는 어머니의 양육불안이 과제를 수행 하는 유아의 자기결정성에 직접적 영향을 미침과 동시에 지시 적.통제적 행동을 매개로 하는 간접적 경로를 통해서도 영향 을 미침을 보여준다. 이는 불안한 어머니는 자녀가 환경에서 자유롭게 탐색할 기회를 제한하거나 통제하는 행동을 통해 의 존감을 심어준다는 선행연구(Becker et al., 2010)와 같은 맥락 에서 논의될 수 있다. 양육불안감이 높은 어머니는 자신의 불 안감으로 인해 자녀가 과제를 할 때 간섭하고 통제하는 행동 을 택하게 되며, 이는 결과적으로 유아의 자기결정성 발달에 부정적 영향을 미칠 수 있다.

이 연구에서는 유아의 자기결정성과 관련이 있는 변인으로 서 어머니의 양육불안과 어머니의 과제 지원 행동을 살펴보고 세 변인의 통합적인 관계를 검증하였으나 다음과 같은 제한점 을 가지고 있다. 첫째, 이 연구는 중산층 가정을 대상으로 진행 되었기 때문에 연구결과를 일반화시키기 어렵다. 후속 연구에 서는 사회인구학적 배경이 다른 참여자를 모집하여 연구대상 의 다양성을 확보할 필요가 있다. 둘째, 이 연구는 자연스러운 일상이나 놀이 상황이 아닌 특수한 상황 즉, 인지적 과제 수행 상황에서의 유아 자기결정성과 어머니의 지원 행동을 살펴보 았기 때문에 연구 결과를 해석하고 반영하는 것에 있어 주의 가 필요하다. 셋째, 선행연구에서 자기결정성은 자아존중감이 나 자기조절력에 의해서도 설명됨을 밝히고 있다(Park \& Seo, 2013). 따라서 추후 연구에서는 유아의 개인 변인을 통제한 상 태에서 환경의 영향력을 더욱 면밀히 살펴볼 필요가 있다.

이러한 제한점에도 불구하고 이 연구는 다음과 같은 의의 
를 갖는다. 첫째, 부모나 교사의 평가로 보고되는 설문지를 사 용하지 않고, 관찰연구법을 통해 유아의 자기결정성과 어머니 의 과제 지원 행동을 측정하여 자료의 신뢰성을 확보하였다는 데 의의가 있다. 둘째, 이 연구는 자기결정성 발달에 있어 유아 기의 중요성에 주목하고 있으며, 아직까지 국내에서 잘 연구 되지 않은 유아를 대상으로 연구하였으며 유아를 둘러싼 가장 중요한 환경 중 하나인 어머니 변인에 중점을 두고 유아의 발 달적 적응에 기여하는 어머니의 역할을 제시하였다는 데 의의 가 있다. 셋째, 이 연구는 우울에 비해 상대적으로 연구가 덜 이루어진 어머니의 양육불안에 대해 조명하였다. 특히 자녀의 학령기 전이를 앞두고 불안감을 경험하는 어머니를 대상으로 하였으며, 자녀 양육의 책임이 어머니에게 부과되고 자녀와의 일체감 의식이 강한 한국의 문화 특성이 고려된 불안감을 중 점적으로 다루었다. 마지막으로 이 연구는 유아의 자기결정성 에 영향을 미치는 어머니의 양육불안과 과제 지원 행동을 함 께 고려함으로써 심리적.행동적 접근을 동시에 모색하고, 이 들 간의 관계를 통합적으로 설명하였다.

\section{Conflict of Interest}

No potential conflict of interest relevant to this article was reported.

\section{Ethics Statement}

All procedures of this research were reviewed by IRB (1910/003010).

\section{References}

\section{In English}

Barrett, K. C., \& Morgan, G. A. (1995). Continuities and discontinuities in mastery motivation during infancy and toddlerhood: A conceptualization and review. In R. H. MacTurk \& G. A. Morgan (Eds.), Mastery motivation: Origins, conceptualizations, and applications (Vol. 12, pp. 57-94). Norwood, NJ: Ablex.

Becker, K. D., Ginsburg, G. S., Domingues, J., \& Tein, J. Y. (2010). Maternal control behavior and locus of control: Examining mechanisms in the relation between maternal anxiety disorders and anxiety symptomatology in children. Journal of Abnormal Child Psychology, 38, 533-543. doi:10.1007/ s10802-010-9388-z

Belsky, J. (1984). The determinants of parenting: A process model. Child Development, 83-96. doi:10.2307/1129836

Bronstein, P., Ginsburg, G. S., \& Herrera, I. S. (2005). Parental predictors of motivational orientation in early adolescence: A longitudinal study. Journal of Youth and Adolescence, 34, 559-575. doi: 10.1007/s10964-005-8946-0

Brotherson, M. J., Cook, C. C., Erwin, E. J., \& Weigel, C. J. (2008). Understanding self-determination and families of young children with disabilities in home environments. Journal of Early Intervention, 31(1), 22-43. doi:10.1177/1053815108324445

Carlton, M. P., \& Winsler, A. (1998). Fostering intrinsic motivation in early childhood classrooms. Early Childhood Education Journal, 25(3), 159-166.

Cha, K. (2015). Associations among children's negative emotionality, executive functions and performance on a cognitive task, and parenting among korean preschoolers (Unpublished doctoral dissertation). Stanford University, Stanford, CA.

Coleman, P. K., \& Karraker, K. H. (2003). Maternal self-efficacy beliefs, competence in parenting, and toddlers' behavior and developmental status. Infant Mental Health Journal, 24(2), 126-148. doi:10.1002/imhj.10048

Cumberland-Li, A., Eisenberg, N., Champion, C., Gershoff, E., \& Fabes, R. A. (2003). The relation of parental emotionality and related dispositional traits to parental expression of emotion and children's social functioning. Motivation and Emotion, 27, 27-56. doi:10.1023/A:1023674308969

Deci, E. L., \& Ryan, R. M. (1985). The general causality orientations scale: Self-determination in personality. Journal of Research in Personality, 19(2), 109-134. doi:10.1016/00926566(85)90023-6

Deci, E. L., \& Ryan, R. M. (1991). A motivational approach to self: Integration in personality. In R. A. Dienstbier (Ed.), Perspectives on motivation (Vol. 38, pp. 237-288). Lincoln, NE: University of Nebraska Press.

Deci, E. L., \& Ryan, R. M. (2000). The "what" and "why" of goal pursuits: Human needs and the self-determination of behavior. Psychological inquiry, 11(4), 227-268. doi:10.1207/ S15327965PLI1104_01

Demertzis, K. H. (2007). The relationship between maternal anxiety and parenting behaviors of negativity and intrusiveness (Unpublished doctoral dissertation). University of California, Los Angeles.

Demertzis, K. H.(2007). The relationship between maternal anxiety and parenting behaviors of negativity and intrusiveness (Unpublished doctoral dissertation). University of California, Los Angeles. 
Diener, M. L., \& Kim, D.-Y. (2004). Maternal and child predictors of preschool children's social competence. Journal of Applied Developmental Psychology, 25(1), 3-24. doi:10.1016/ j.appdev.2003.11.006

Farkas, M. S., \& Grolnick, W. S. (2010). Examining the components and concomitants of parental structure in the academic domain. Motivation and Emotion, 34, 266-279. doi:10.1007/s11031-010-9176-7

Ginsburg, G. S., Grover, R. L., \& Ialongo, N. (2005). Parenting behaviors among anxious and non-anxious mothers: Relation with concurrent and long-term child outcomes. Child \& Family Behavior Therapy, 26(4), 23-41. doi:10.1300/J019v26n04_02

Ginsburg, G. S., Grover, R. L., Cord, J. J., \& Ialongo, N. (2006). Observational measures of parenting in anxious and nonanxious mothers: Does type of task matter? Journal of Clinical Child \& Adolescent Psychology, 35(2), 323-328. doi:10.1207/ s15374424jccp3502_16

Ginsburg, G. S., \& Schlossberg, M. C. (2002). Family-based treatment of childhood anxiety disorders. International Review of Psychiatry, 14(2), 143-154. doi:10.1080/09540260220132662

Gottfried, A. E., Fleming, J. S., \& Gottfried, A. W. (1994). Role of parental motivational practices in children's academic intrinsic motivation and achievement. Journal of Educational Psychology, 86(1), 104-113. doi:10.1037/0022-0663.86.1.104

Grolnick, W. S., \& Ryan, R. M. (1989). Parent styles associated with children's self-regulation and competence in school. Journal of Educational Psychology, 81(2), 143-154. doi:10.1037/00220663.81.2.143

Grolnick, W. S., Raftery-Helmer, J. N., Marbell, K. N., Flamm, E. S., Cardemil, E. V., \& Sanchez, M. (2014). Parental provision of structure: Implementation and correlates in three domains. Merrill-Palmer Quarterly, 60(3), 355-384. doi:10.13110/merrpalmquar1982.60.3.0355

Grolnick, W. S., Ryan, R. M., \& Deci, E. L. (1991). Inner resources for school achievement: Motivational mediators of children's perceptions of their parents. Journal of Educational Psychology, 83(4), 508-517. doi:10.1037/00220663.83.4.508

Hallion, L. S., \& Ruscio, A. M. (2011). A meta-analysis of the effect of cognitive bias modification on anxiety and depression. Psychological Bulletin, 137(6), 940-958. doi:10.1037/ a0024355

Harackiewicz, J. M., Manderlink, G., \& Sansone, C. (1984). Rewarding pinball wizardry: Effects of evaluation and cue value on intrinsic interest. Journal of Personality and Social Psychology, 47(2), 287-300. doi:10.1037/0022-3514.47.2.287

Hayes, A. F. (2013). Introduction to mediation, moderation, and conditional process analysis: A regression-based approach. New York: Guilford Press.

Hoffman, C., Crnic, K. A., \& Baker, J. K. (2006). Maternal depression and parenting: Implications for children's emergent emotion regulation and behavioral functioning. Parenting: Science and Practice, 6(4), 271-295. doi:10.1207/s15327922par0604_1

Hudson, J. L., \& Rapee, R. M. (2001). Parent-child interactions and anxiety disorders: An observational study. Behaviour Research and Therapy, 39(12), 1411-1427. doi:10.1016/ S0005-7967(00)00107-8

Lepper, M. R., \& Greene, D. (1975). Turning play into work: Effects of adult surveillance and extrinsic rewards on children's intrinsic motivation. Journal of Personality and Social Psychology, 31(3), 479-486. doi:10.1037/h0076484

Levesque, C., Zuehlke, A. N., Stanek, L. R., \& Ryan, R. M. (2004). Autonomy and competence in German and American university students: A comparative study based on selfdetermination theory. Journal of Educational Psychology, 96(1), 68-84. doi:10.1037/0022-0663.96.1.68

Moore, P. S., Whaley, S. E., \& Sigman, M. (2004). Interactions between mothers and children: Impacts of maternal and child anxiety. Journal of Abnormal Psychology, 113(3), 471476. doi:10.1037/0021-843X.113.3.471

Neitzel, C., \& Dopkins Stright, A. (2004). Parenting behaviours during child problem solving: The roles of child temperament, mother education and personality, and the problem-solving context. International Journal of Behavioral Development, 28(2), 166-179. doi:10.1080/01650250344000370

Pettit, G. S., Laird, R. D., Dodge, K. A., Bates, J. E., \& Criss, M. M. (2001). Antecedents and behavior-problem outcomes of parental monitoring and psychological control in early adolescence. Child Development, 72(2), 583-598. doi:10.1111/1467-8624.00298

Roth, G., Assor, A., Niemiec, C. P., Ryan, R. M., \& Deci, E. L. (2009). The emotional and academic consequences of parental conditional regard: Comparing conditional positive regard, conditional negative regard, and autonomy support as parenting practices. Developmental Psychology, 45(4), 1119-1142. doi:10.1037/a0015272

Rubin, K. H., Coplan, R. J., \& Bowker, J. C. (2009). Social withdrawal in childhood. Annual Review of Psychology, 60, 141-171. doi:10.1146/annurev.psych.60.110707.163642

Ryan, R. M., \& Deci, E. L. (2000). Intrinsic and extrinsic motivations: Classic definitions and new directions. Contemporary Educational Psychology, 25(1), 54-67. doi:10.1006/ceps.1999.1020

Ryan, R. M., \& Lynch, J. H. (1989). Emotional autonomy versus detachment: Revisiting the vicissitudes of adolescence and young adulthood. Child Development, 60(2), 340-356. doi: $10.2307 / 1130981$

Ryan, R. M., Huta, V., \& Deci, E. L. (2008). Living well: A selfdetermination theory perspective on eudaimonia. Journal of Happiness Studies, 9, 139-170. doi:10.1007/s10902-006- 
9023-4

Schunk, D. H. (1991). Self-efficacy and academic motivation. Educational Psychologist, 26(3-4), 207-231.

Spielberger, C. D. (1972). Anxiety: Current trends in theory and research. New York: Academic Press.

Stevenson-Hinde, J., Shouldice, A., \& Chicot, R. (2011). Maternal anxiety, behavioral inhibition, and attachment. Attachment \& Human Development, 13(3), 199-215. doi:10.1080/146 16734.2011.562409

Tafarodi, R. W., Milne, A. B., \& Smith, A. J. (1999). The confidence of choice: Evidence for an augmentation effect on selfperceived performance. Personality and Social Psychology Bulletin, 25(11), 1405-1416. doi:10.1177/0146167299259006

Trapolini, T., McMahon, C. A., \& Ungerer, J. A. (2007). The effect of maternal depression and marital adjustment on young children's internalizing and externalizing behaviour problems. Child: Care, Health and Development, 33(6), 794-803. doi:10.1111/j.1365-2214.2007.00739.x

Turner, S. M., Beidel, D. C., Roberson-Nay, R., \& Tervo, K. (2003). Parenting behaviors in parents with anxiety disorders. Behaviour Research and Therapy, 41(5), 541-554. doi:10.1016/S0005-7967(02)00028-1

Vansteenkiste, M., Sierens, E., Goossens, L., Soenens, B., Dochy, F., Mouratidis, A.,...Beyers, W. (2012). Identifying configurations of perceived teacher autonomy support and structure: Associations with self-regulated learning, motivation and problem behavior. Learning and Instruction, 22(6), 431-439. doi:10.1016/j.learninstruc.2012.04.002

Whaley, S. E., Pinto, A., \& Sigman, M. (1999). Characterizing interactions between anxious mothers and their children. Journal of Consulting and Clinical Psychology, 67(6), 826836. doi:10.1037/0022-006X.67.6.826

Woodruff-Borden, J., Morrow, C., Bourland, S., \& Cambron, S. (2002). The behavior of anxious parents: Examining mechanisms of transmission of anxiety from parent to child. Journal of Clinical Child and Adolescent Psychology, 31(3), 364-374. doi:10.1207/S15374424JCCP3103_08

Zhang, H., \& Whitebread, D. (2019). Examining Chinese kindergarten children's psychological needs satisfaction in problem solving: A self-determination theory perspective. Instructional Science, 47, 373-398. doi:10.1007/s11251019-09490-5

\section{In Korean}

Choi, B.-Y., \& Lew, K.-H. (2010). The structural relationship among the self-determination motivation, self-regulated learning, and academic achievement in elementary school. Journal of Education \& Culture, 16(3), 183-203. doi:10.24159/joec.2010.16.3.183
Ju, H.-J. (2011). Analysis of the causal relationships among selfdetermination motivation, self-directed learning ability, academic self-efficacy and academic achievement of elementary school students. Journal of Learner-Centered Curriculum and Instruction, 11(2), 237-259.

Kang, S.-K., Chung, M.-R., Yee, B.-S., \& Kim, M.-J. (2014). A comparison study of parenting involvement, efficacy and anxiety of Korean and French mothers. Korean Journal of Early Childhood Education, 34(6), 539-555. doi:10.18023/ kjece.2014.34.6.023

Kim, A., \& Doh, H.-S. (2018). Transactional relationships among children's negative emotionality, mothers' depression, and parenting behavior. Korean Journal of Child Studies, 39(3), 45-59. doi:10.5723/kjcs.2018.39.3.45

Kim, M., \& Shin, T. (2010). Test of a structural model among school's psychological environments, self-determination, self-regulated behavior, student achievement, and school satisfaction. Asian Journal of Education, 11(3), 43-70.

Kim, Y. J. (2018). The relationship of preschoolers' self-determination, self-esteem, level of home observation for measurement of the environment, and goodness of fit between motherchild according to household income level (Master's thesis). Retrieved from http://www.riss.kr/link?id=T14760748

Kong, B., \& Baek, S. (2017). The influence of parenting experience on parenting anxiety and parental satisfaction-Focusing on comparison between Korea and Japan-. Korean Journal of the Japan Education, 22(1), 87-105.

Lee, H., \& Park s.-Y. (2020). The mediating effects of child self-determination between child-perceived mother's parenting behavior and child subjective well-being. Korean Association for Play Therapy, 23(2), 163-180. doi:10.17641/ KAPT.23.2.4

Lee, J., \& Jang, M. (2009). Relationship between maternal depression and parenting behaviors: The mediating effects of irrational beliefs in parenting. Cognitive Behavior Therapy in Korea, 9(2), 1-16.

Lee, Y.-S. (2017). Impact of perceived propensities for depression and anxiety on maternal style in mothers with children in early childhood. Korean Journal of Local Government \& Administration Studies, 31(4), 309-335.

Lim, H.-J. (2019). A study on effects of mothers' parental competence, mothers' parental anxiety, child's self-esteem, and child's happiness on child's social competence. The Journal of Korea Open Association for Early Childhood Education, 24(5), 133156. doi:10.20437/KOAECE24-5-07

Ministry of Education. (2019). Kindergarten curriculum notice (Report No. 2019-189). Retrieved from Ministry of Education website: www.moe.go.kr

Noh, B.-H., Park, S.-Y., Chee, Y. K. (2011). The relationship between socioeconomic status, maternal involvement 
in learning, parenting behavior and children's selfdetermination motivation. Korean Journal of Child Studies, 32(4), 83-97. doi:10.5723/KJCS.2011.32.4.83

Park, G. J., \& Seo, S. J. (2013). Determinants of preschoolers' selfdetermination: Focus on their playfulness, self-esteem, and self-regulation. Journal of Korean Child Care and Education, 9(5), 489-511.

Statistics Korea. (2020). 2020 1/4bungi gagyedonghyangjosa gyeolgwa [2020 1/4분기 가계동향조사 결과]. Retrieved June 21, 2020, from http://kostat.go.kr/

Sung, H. Y., Bang, H. J., \& Lee, S. H. (2005). The effects of mother behavior characteristics and attachment security on infant behavior characteristics in problem solving situation. The Korean Journal of Development Psychology, 18(2), 57-74.

We, J.-H. (2014). Development of the parenting anxiety scale and its validation study (Doctoral dissertation). Retrieved from http://www.riss.kr/link?id=T13391188
Yeo, T. (2015). The moderating effect of children's optimism and self-determination in the relation between academic stress and school adjustment. The Korean Journal of Elementary Counseling, 14(1), 25-42.

\section{ORCID}

Hyun A Lee https://orcid.org/0000-0002-0733-1772

Kangyi Lee https://orcid.org/0000-0002-1193-5771

Received December 30, 2020

Revision received February 5, 2021

Accepted February 8, 2021 